\title{
Integrating Behavioral Health into Primary Care
}

\author{
Peter M. McGough, MD, Amy M. Bauer, MD, MS, \\ Laura Collins, LICSW, ${ }^{3}$ and David C. Dugdale, MD, FACP ${ }^{4}$
}

\begin{abstract}
Depression is one of the more common diagnoses encountered in primary care, and primary care in turn provides the majority of care for patients with depression. Many approaches have been tried in efforts to improve the outcomes of depression management. This article outlines the partnership between the University of Washington (UW) Neighborhood Clinics and the UW Department of Psychiatry in implementing a collaborative care approach to integrating the management of anxiety and depression in the ambulatory primary care setting. This program was built on the chronic care model, which utilizes a team approach to caring for the patient. In addition to the patient and the primary care provider (PCP), the team included a medical social worker (MSW) as care manager and a psychiatrist as team consultant. The MSW would manage a registry of patients with depression at a clinic with several PCPs, contacting the patients on a regular basis to assess their status, and consulting with the psychiatrist on a weekly basis to discuss patients who were not achieving the goals of care. Any recommendation (eg, a change in medication dose or class) made by the psychiatrist was communicated to the PCP, who in turn would work with the patient on the new recommendation. This collaborative care approach resulted in a significant improvement in the number of patients who achieved care plan goals. The authors believe this is an effective method for health systems to integrate mental health services into primary care. (Population Health Management 2016;19:81-87)
\end{abstract}

\section{Introduction}

D EPRESSION REMAINS ONE OF the most common mental health conditions in the United States, with between 13.1 and 14.2 million patients experiencing an episode of major depression each year. ${ }^{1}$ The overall disease burden from major depression has increased $43 \%$ between 1990 and 2010, such that it is now the second leading cause of disability and the fifth leading cause of overall disease burden in the United States. ${ }^{2}$ Fewer than $20 \%$ of depressed patients are seen by a psychiatrist or psychologist; the majority of these patients are seen in primary care settings. ${ }^{3}$ Despite attempts at medication treatment and occasional referral for specialty consultation, only about $25 \%$ of patients improve. ${ }^{4}$ Although many of these nonresponders may be thought to have treatment-resistant depression, it is likely that the legacy approach to the management of these patients is a significant part of the problem. ${ }^{5}$ The Triple Aim proposed for accountable care (better outcomes, better patient experience, and lower cost) is a stimulus for examining new approaches to the management of chronic health conditions including models of collaborative care.

The traditional approach taken by most primary care providers (PCPs) in diagnosing and managing anxiety and depression has been visit based and problem centered. Most often, the diagnosis has been considered when a patient raised the concern ("I think I may be depressed") or occasionally when a patient with another chronic condition (eg, diabetes, heart failure) was not responding to or complying with treatment recommendations. Efforts to screen the broader population have been uncommon, in part because the US Preventive Services Task Force recommends screening only if there is a system of care in place for managing depression. ${ }^{6}$ Trials of treatment with medication were often limited in time and scope, with sporadic contact with the patient and high patient drop-out rates being the rule. There was not wide acceptance of quantitative, evidence-based tools (eg, the Patient

${ }^{1}$ UW Medicine Neighborhood Clinics, and Department of Family Medicine, Seattle, Washington.

${ }^{2}$ Department of Psychiatry and Behavioral Sciences, University of Washington, Seattle, Washington.

${ }^{3}$ Psychiatry and Behavioral Sciences, PCS, University of Washington, Seattle, Washington.

${ }^{4}$ Care Management and Population Health, UW Medicine, and Department of Medicine, UW School of Medicine, University of Washington.

(c) The Author(s) 2015; Published by Mary Ann Liebert, Inc. This Open Access article is distributed under the terms of the Creative Commons Attribution Noncommercial License (http://creativecommons.org/licenses/by-nc/4.0/) which permits any noncommercial use, distribution, and reproduction in any medium, provided the original author(s) and the source are credited. 
Health Questionnaire-9 [PHQ-9]) in managing depressed patients to assess response to treatment, instead relying on more subjective and global assessments of patient status. This is akin to physicians attempting to manage patients with hypertension without routinely checking blood pressure. Finally, there was rarely an attempt to "treat to goal," with most patients and physicians accepting subtherapeutic regimens as "good enough," which has been described as clinical inertia. ${ }^{5}$

Over time, many PCPs developed relationships with mental health specialists in their community and would refer patients who they felt were challenging or doing poorly. These referrals were often sporadic and poorly coordinated because the PCP and mental health providers utilized different health records and approaches to care. This was further complicated by challenges of limited access to scarce mental health consultants. ${ }^{7}$ In this confusing maze, many patients simply "fell through the cracks." The net result of inadequacies in depression care is that few patients improve: fewer than $20 \%$ of patients started on antidepressant medications in usual primary care show substantial clinical improvements, ${ }^{8,9}$ and patients referred to psychotherapy often receive inadequate trials of such treatments and/or ineffective forms of psychotherapy, so that treatment response for this type of treatment is also as low as $20 \%$ under usual care. ${ }^{10}$

One approach to addressing these shortcomings involved "colocating" mental health specialists in primary care settings. Even with this improved access and decreased fragmentation, there were still the problems of nonsystematic referrals, often with high no-show rates. Despite the intuitive attraction of colocation, there is a lack of evidence to support this approach to improving outcomes for depressed patients. $^{11}$

Over the past 2 decades, many health plans and selfinsured businesses have taken the approach of "carving out" mental health services with the goal of limiting access to a small number of providers who follow agreed-upon treatment protocols. This form of utilization management created further fragmentation of mental from physical health care, often to the detriment of both. Although there may have been some reduction of direct mental health costs, this approach did not address the wider costs and impacts of mental health issues on patients and the community, as will be outlined.

Despite improved awareness of common mental disorders such as anxiety and depression among PCPs, these conditions remain underdiagnosed and undertreated with substantial repercussions for patients and society. Depression starts earlier in life and has a greater impact on quality of life and functioning than many chronic medical conditions. Depression also is associated with

- Health risk behaviors such as smoking, inactivity, obesity, and substance use;

- Increased risk for chronic diseases and their complications;

- Poorer adherence to medical management;

- Worse medical outcome;

- Increased health care costs largely attributable to use of acute medical services and early mortality. ${ }^{12}$

In addition to increased cost burden to the medical system and society, patients with depression and other mental health conditions may be considered to be "difficult" by PCPs. The collaborative care model, which will be described, exemplifies how such a clinical care model can contribute to health systems' efforts to achieve the Triple Aim. ${ }^{13}$

The chronic care model was developed by Wagner and colleagues nearly 2 decades ago to guide the reorganization of health services for more effective management of chronic diseases. ${ }^{14}$ The model proposes that improved patient outcomes result from the interactions between an activated patient and a proactive health care team, with a number of factors facilitating patient activation and preparation of the health care team. For patients, these factors include support for disease self-management and enabling community resources. For health care providers, these factors include adequate information systems, decision support, and delivery system design to support longitudinal care as opposed to episodic care.

Collaborative care is a model of care that applies the chronic disease model to the treatment of common mental disorders, notably depressive and anxiety disorders. Evidence for the collaborative care model is robust, with support for its effectiveness from more than 80 randomized trials over the last 2 decades. ${ }^{15,16}$ Data come from diverse settings across patient age ranges, socioeconomic and racial/ ethnic groups, and a variety of mental health conditions and medical comorbidities (eg, diabetes, cardiac disease, cancer). ${ }^{17}$ The scalability of the practice model is supported by several large programs including Washington State's Mental Health Integration Program, the DIAMOND program in Minnesota, and in the Department of Defense. ${ }^{10}$ Cost savings also have been realized in real-world models that integrate behavioral health and primary care. ${ }^{18}$

With decades of research evidence and more than 80 randomized trials supporting the effectiveness of collaborative care for management of a variety of common mental disorders in primary care, attention has shifted toward the need to promote implementation of this evidence-based model of care. $^{13,19}$ To address the knowledge gap related to successful implementation of collaborative care, this article describes the implementation and evaluation of a collaborative care program in an academic-affiliated primary care system, including key barriers and facilitators, program outcomes, lessons learned, and recommendations for other systems that are considering implementing collaborative care.

\section{Methods}

\section{The Behavioral Health Integration Program (BHIP) model of care}

Collaborative care is provided by a primary care-based team that includes the PCP, a care manager (a role that can be filled by a clinical social worker, registered nurse [RN], or psychologist, among others), and a psychiatric consultant. PCPs serve as the initial point of contact for patients, diagnose patients with mental health conditions such as depression or anxiety, and retain a key role and responsibility in overseeing the coordinated care provided by the team. Care managers work closely with the PCPs and perform a number of functions, including:

- A structured comprehensive mental health assessment;

- Patient engagement and education;

- Delivery of brief evidence-based behavioral interventions (problem-solving therapy, motivational inter- 
viewing, behavioral activation, cognitive and dialectical behavioral therapy);

- Proactive follow-up to monitor treatment response using standardized instruments with specific goals;

- Weekly caseload review, with a psychiatric consultant, of patients who are not improving as expected;

- Care coordination and facilitation of communication between members of the treatment team; and

- Facilitation of referrals to and coordination with community-based agencies, outside mental health or medical specialty care, substance abuse services, and social services.

A typical full-time care manager carries an active caseload of 50-100 patients and will treat about 150 patients during a year. Most patients treated in collaborative care do not require or receive direct services from the psychiatric consultant. However, for selected patients who do not respond to treatment or are diagnostically complex, the psychiatric consultant may provide a direct patient consultation. This method of psychiatric caseload review supported by direct service provision on a stepped care basis provides an efficient means of leveraging limited specialist resources across a larger patient population. Within the stepped care approach, patient outcomes are systematically monitored using standardized tools (eg, PHQ-9, Generalized Anxiety Disorder scale [GAD-7] $)^{20}$ and treatments are adjusted until the patient achieves the targeted clinical outcome. This treatment-to-target approach is a major reason that collaborative care results in improved patient outcomes. Its implementation is facilitated by the use of a patient registry to track progress and outcomes for all patients initiating care so that no one "falls through the cracks."

\section{Setting}

The UW Neighborhood Clinic (UWNC) network is in the process of achieving Level 3 patient-centered medical home certification. A key requirement of Level 3 certification is having an effective patient care management and coordination infrastructure as well as effectively integrating behavioral health into primary care. UWNC's earliest efforts in care management focused on diabetes and engaging RNs and certified dieticians in working with the clinic's PCPs and patients with diabetes.

\section{Target population}

Primary care patients with depressive or anxiety disorders who are not receiving specialty mental health services are the target population for the BHIP, although there are not rigid criteria for enrollment. Patients are referred to BHIP by PCPs based on the PCP's assessment that the patient has a mental health need that can be served by the program. Consequently, many patients served by BHIP have comorbid medical conditions.

\section{Program implementation}

A pilot program was created at one of the organization's clinics with known high mental health needs. An experienced social worker with master's level training (MSW) who had previously worked at the safety net hospital in the emergency department was recruited. One of the first things the MSW was able to validate was that the patients seen at the pilot clinic site

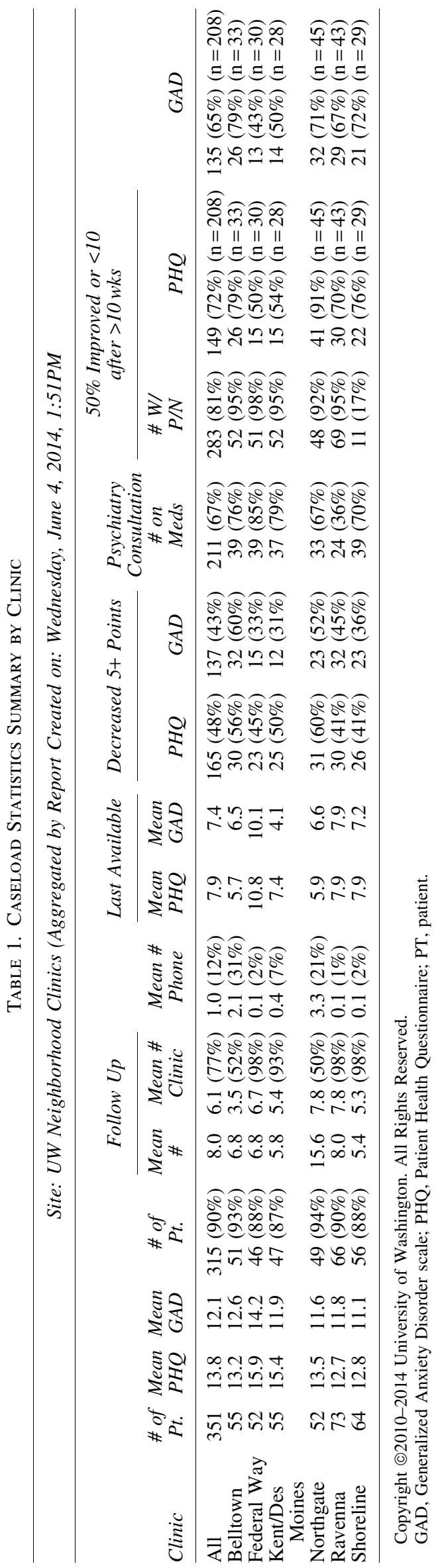




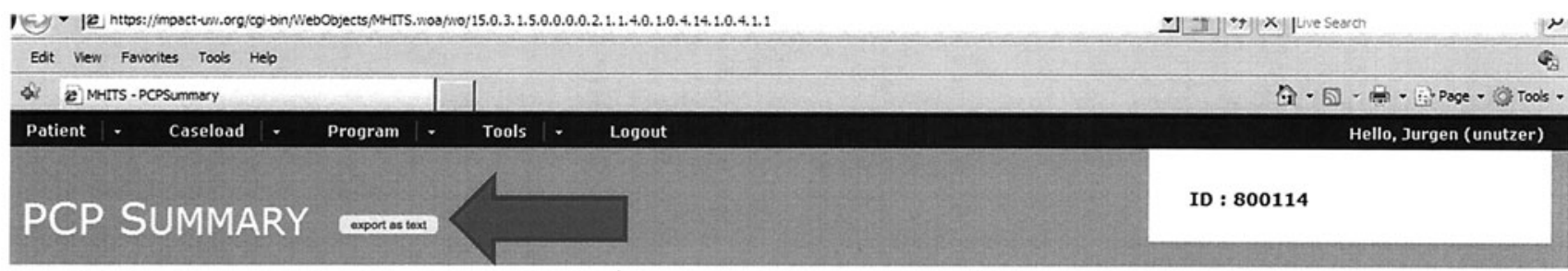

Care Coordinator Created on: Wednesday, February 3, 2010

Working Diagnoses :

L1 : Depression (PHQ-9 : O/27, Minimal); Anxiety (GAD-7:0/21); PTSO (PCL : 56/85)

Formulation : Pt feels significantly better. No depressive sxs and only 'normal' anxiety. States previously her sister had a fight $w$ her mother, pt became estranged from her mother and sister for a time. Pt continues to have a good relationship wher mother and her sister if mending her relationship w the mother. Pt discussed how she would work wher sister. Reports good relationship w her husband whose Treatment Progress :

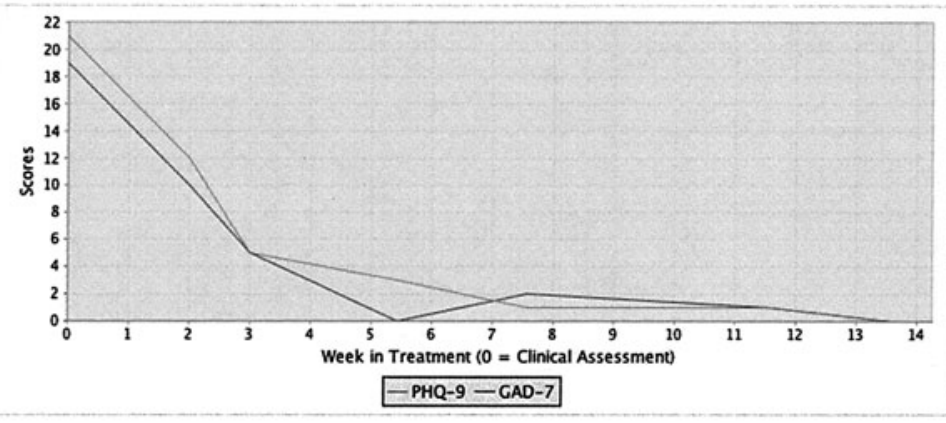

Safety Concers :

Past Suicide Attempts : None reported.

Current Psychiatric Medications : Sertraline (Zoloft) / 50mg, 1 tablet once a day

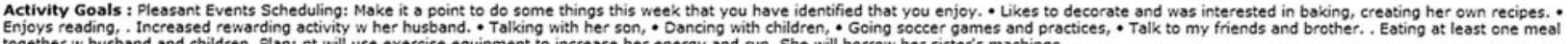
together w husband and children. Flan: pt will use exercise equipment to increase her energy and run. She will borrow her sister's machinge.

Referrals : None recorded

FIG. 1. Summary for the primary care physician (PCP) with graphed depression and anxiety scores.

were not the "worried well" but rather a cohort of patients with moderate to serious mental health diagnoses and needs. After selected staff received training in the BHIP model, a patient registry was developed that included patients with poorly controlled anxiety and depression who were then enrolled in the care management program. Based on initial positive patient outcomes and reviews from both patients and PCPs, the program was expanded beyond the pilot clinic; a care manager and consulting psychiatrist were placed in 6 of the 9 clinics over a period of 1 year, and has since expanded to all 9 clinics in the network during the second year.

\section{Data}

The program uses a Web-based registry, named the Care Manager Tracking System (CMTS, Table 1), to provide a

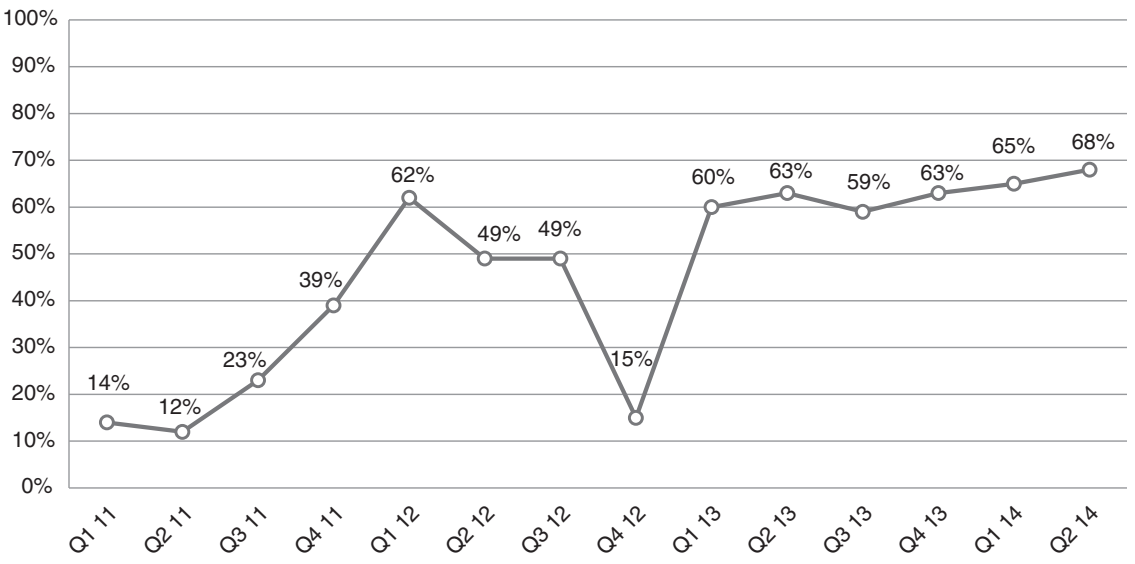

FIG. 2. Percent of active Behavioral Health Integration Program (BHIP) patients with $\geq 2$ monthly contacts per quarter. 
summary of how many patients are meeting quality indicators including: PHQ-9 and GAD- $7^{20,21}$ score improvement (target is a 5-point improvement for at least $45 \%$ of the caseload), psychiatry consultation for patients who are not improving (target is $80 \%$ ), and documentation of psychiatric medications in the registry. The registry also tracks the number of in-clinic and phone follow-up visits and the percentage of the caseload that has demonstrated at least a 50\% improvement in depression or anxiety scores. The care manager or PCP also can also visualize each patient's progress with symptoms, as shown in Figure 1. This allows a consulting psychiatrist to conduct a systematic review of patient participation in the program as well as clinical improvements of patients who are not achieving their treatment goals. This article reports descriptive data obtained from the patient registry for the period from January 2011 to August 2014.

\section{Results}

The overall patient population at the 9 UWNCs is primarily commercially funded $(70 \%$ commercial insurance, $12 \%$ Medicaid, and $12 \%$ Medicare). The average age of the clinic patients is 41 , ranging from newborns to 103 years old. Males comprise $44 \%$ of the patients. The BHIP population ( $\mathrm{n}=1256$ patients as of August 2014) in these clinics primarily presents with depression $(\mathrm{n}=955$ [76\%]) and anxiety $(\mathrm{n}=528[42 \%])$, posttraumatic stress disorder $(\mathrm{n}=188$ [15\%]), with some bipolar disorder $(\mathrm{n}=201[16 \%])$, and alcohol/substance abuse $(\mathrm{n}=151$ [12\%]) (diagnosis categories are not mutually exclusive). Forty percent of the population $(n=502)$ reported thoughts of suicide based on their responses to questions on the PHQ-9.

\section{Patient engagement and clinical outcomes}

The UWNCs have demonstrated steady improvement in engaging patients in care, as indicated by monthly patient contacts. These may be in clinic or over the phone; approximately $76 \%$ of patient contacts take place in clinic. The quality aim for this indicator is defined as: $\geq 2$ patient contacts per month with more than half of the caseload. The UWNC care managers have consistently exceeded this target with more than $60 \%$ of the caseload engaged in biweekly care since 2013 (Fig. 2). With regard to what appears to be a dip in Q4 2012, prior to that time, the program was essentially fully deployed in only 1 clinic. By Q4 of 2012, the BHIP expanded with most of the remaining neighborhood clinics. In turn, the percentage of monthly contacts started low and went up from there.

The number of patients actively served at one time also increased to more than 900 across the entire UWNC during its first year. As of July 2014, on average, the BHIP patients are seen for 8.1 follow-up appointments with the care coordinators (CMTS database) over the course of their treatment in BHIP, reflecting a high level of patient engagement.

In the first year of full program implementation, more than $45 \%$ of the BHIP caseload demonstrated at least a 5point drop in depression or anxiety scores (Table 2). This improvement has been maintained, with more than $60 \%$ of the overall caseload demonstrating significant improvement from 2013 to 2014. In addition, more than 70\% (PHQ-9) and $65 \%$ (GAD-7) of the BHIP population has demonstrated a $50 \%$ improvement in symptoms (or scoring $<10$ - mild symptomology) after at least 10 weeks in treatment. These outcomes also have stayed consistent from 2014 to the date of this paper.

\section{Patient access}

Between January 2011 and August 2014 there were 1256 total patients enrolled in the BHIP at the UWNC sites. The program has discharged 788 of these patients after achieving treatment goals; 348 patients are currently enrolled. In 2010, only 1 of the UWNCs offered mental health care in its clinic, which was limited to only psychotherapy. With the BHIP implementation since November 2012, a secondary outcome of the BHIP was that improved care management of behavioral health patients allowed many PCPs to serve additional patients.

\section{Program costs}

This program leverages a scarce, expensive resource (the psychiatrist) with the use of a less expensive provider (care manager). This allows for better health outcomes and consistent care using a lower cost provider. This results in providing a higher value of care.

While the research team was not able to assess the total cost impact of the BHIP directly, they were able to estimate it by using data from the IMPACT Study, which looked at the difference in outpatient and inpatient costs (including hospital admissions and emergency department use) as a result of introducing a coordinated care approach to behavioral health. ${ }^{22}$ The team estimated that over a 4-year

Table 2. Behavioral Health Integration Program Outcomes Summary

\begin{tabular}{lccc}
\hline Indicator & Target & At 1 year (1/1/2014) & Current (August 14) \\
\hline Total \# of patients enrolled over time & As of 1/1/13: 273 & 911 & 1256 \\
Mean care manger caseload (0.5 FTE) & 50 & 53 & 52 \\
5 point improvement in either depression or anxiety & $50 \%$ & $44.5 \%$ & $57.3 \%$ \\
$50 \%$ improved with depression or score under 10 after 10+ & $50 \%$ & $76 \%$ & $70 \%$ \\
$\quad$ weeks in treatment & $50 \%$ & $69 \%$ & $64 \%$ \\
$50 \%$ improved with anxiety or score under 10 after 10+ & & $\sim \$ 750,000$ & 2 year Projection: \\
$\quad$ weeks in treatment & & & $\sim \$ 1,500,000$ \\
\hline
\end{tabular}

FTE, full-time employee. 
period there would be an overall savings of $\$ 3363$ per patient, yielding a return on investment of $\$ 6.5$ saved for each $\$ 1$ invested. $^{22}$ As of August 14, 2014 more than 1200 patients had been enrolled in the program, yielding a 2-year projected cost savings of $\$ 1,500,000$. Although the latter was not realized as a financial gain to the clinics in the existing fee-for-service environment, the value of this was recognized within the accountable care environment, for which the organization is planning.

\section{Discussion}

Utilizing a collaborative care approach and a chronic disease model, the BHIP embedded within UWNC resulted in good outcomes of care for patients with anxiety and depression that are comparable to or exceed the rates of improvement realized in clinical trials. ${ }^{23}$ Although there is value in better management of patients who have only anxiety and/or depression, the impact on the care of patients who have medical conditions and mental health comorbidities is also significant by creating more effective patient engagement and activation.

Several limitations of this program evaluation should be acknowledged. There were no data on patient outcomes prior to implementing the program; therefore, published rates of improvement from the literature were used as a comparator. Second, although the program met metrics for care processes and patient improvement, the evaluation was not designed to address the question of which specific care processes may have led to patient improvement. Finally, data on costs were estimated based on the literature.

Although this program was fortunate to have great results from the very beginning, there are some "lessons learned" that may help other health systems who seek to implement similar programs. First, it was critical to communicate a clear vision (the Why) to everyone involved. This is especially important for the PCPs who refer patients to the program. The research team found it effective to have a lead psychiatrist meet with the clinic PCPs as a group and explain the program in detail. This was not a program to "off-load" the most challenging patients, and not all patients were appropriate for the program (eg, those with complex mental health diagnoses or some personality disorders). It was critical to have PCP buy-in, and in turn to have them introduce the program to their patients in a positive way. They needed to be clear that they were not "sending them away," but bringing in more resources in support of patient care.

Next, it was important to have an infrastructure that included information technology tools to support registries and tracking of patients and metrics. Because most information technology systems are constantly changing, making sure the necessary tools and reports are maintained is important.

Effective recruitment and training of care managers was essential. They needed to have strong communication skills, the ability to think on their feet, and to work effectively in a team environment. It also was critical to make sure the care managers and consulting psychiatrists were not overwhelmed with other more traditional tasks and consults, allowing them to focus on the care management program.

Operationally, it helped to have a strong pilot site. This allowed for recognition of early wins and developed strong champions for the program as it spread. This made more widespread implementation of the program easier.

As a primary care network also affiliated with a large academic health system, the BHIP was used to train residents within interdisciplinary teams wherein all members work at the top of their scope of practice. This has been true for both primary care and specialty care trainees, and in the future the research team hopes to engage other trainees (eg, $\mathrm{RN}$ and MSW students, psychiatry residents and fellows) in team care.

Finally, it was very important to report the results of the program regularly, both to clinic providers and staff and to health system leadership. This helped reinforce support for the program and energized those involved in continuing the work. The research team believes that this new integrated approach to behavioral health will strengthen primary care as well.

\section{Author Disclosure Statement}

Drs. McGough, Bauer, Dugdale, and Ms. Collins declared no conflicts of interest with respect to the research, authorship, and/or publication of this article. The authors received no financial support for the research, authorship, and/ or publication of this article.

\section{References}

1. Kessler RC, Berglund P, Demler O, et al. The epidemiology of depressive disorder; results from the National Comorbidity Survey Replication (NCS-R). JAMA. 2003; 289:3095-3105.

2. Institute for Health Metrics and Evaluation (IHME). The State of US Health: Innovations, Insights, and Recommendations from the Global Burden of Disease Study. Seattle, WA: IHME; 2013.

3. Wang PS, Demler O, Olfson M, Pincus HA, Wells KB, Kessler RC. Changing profiles of service sectors used for mental health care in the United States. Am J Psychiatry. 2006;163:1187-1198.

4. Wang PS, Lane M, Olfson M, Pincus HA, Wells KB, Kessler RC. Twelve-month use of mental health services in the United States: results from the National Comorbidity Survey Replication. Arch Gen Psychiatry. 2005;62:629-640.

5. Henke RM, Zaslavsky AM, McGUire TG, Ayanian JZ, Rubenstein LV. Clinical inertia in depression treatment. Med Care. 2009;47:959-967.

6. U.S. Preventive Services Task Force. Screening for depression in adults: U.S. Preventive Services Task Force recommendation statement. Ann Intern Med. 2009;151:784-792.

7. Cunningham PJ. Beyond parity: primary care physicians' perspectives on access to mental health care. Health Aff (Millwood). 2009;28(3):w490-w501.

8. Rush AJ, Trivedi M, Carmody TJ, et al. One-year clinical outcomes of depressed public sector outpatients: A benchmark for subsequent studies. Biol Psychiatry. 2004; 56(1):46-53.

9. Unützer J, Katon W, Callahan M, et al. Collaborative care management of late-life depression in the primary care setting. JAMA. 2002;288:2836-2845.

10. Hansen N. The psychotherapy dose-response effect and its implications for treatment delivery services. Clin PsycholSci Pr. 2002;9:329-343.

11. Thielke $S$, Vannoy $S$, Unützer J. Integrating mental health and primary care. Prim Care. 2007;34:571-592, vii. 
12. Katon W. Clinical and health services relationships between major depression, depressive symptoms, and general medical illness. Biol Psychiatry. 2003;54:216-226.

13. Katon WJ, Unützer J. Health reform and the Affordable Care Act: the importance of mental health treatment to achieving the triple aim. J Psychosom Res. 2013;74:533-537.

14. Wagner EH. Chronic disease management: what will it take to improve care for chronic illness? Eff Clin Pract. 1998; 1(1):2-4.

15. Archer J, Bower P, Gilbody S, et al. Collaborative care for depression and anxiety problems. Cochrane Database Syst Rev. 2012;10:CD006525.

16. Thota AB, Sipe TA, Byard GJ, et al. Collaborative care to improve the management of depressive disorders: a community guide systematic review and meta-analysis. Am J Prev Med. 2012;42:525-538.

17. Substance Abuse and Mental Health Services Administration. The President's New Freedom Commission on Mental Health. Achieving the Promise: Transforming Mental Health Care in America. 2003. http://store.samhsa.gov/product/ Achieving-the-Promise-Transforming-Mental-Health-Carein-America-Executive-Summary/SMA03-3831. Accessed June 16, 2015.

18. Reiss-Brennan B, Briot PC, Savitz LA, Cannon W, Staheli R. Cost and quality impact of Intermountain's mental health integration program. Journal of Healthcare Management. 2010;55(2):1-18.
19. Katon W, Unützer J. Collaborative care models for depression: time to move from evidence to practice. Arch Intern Med. 2006;166:2304-2306.

20. Kroenke K, Spitzer RL, Williams JB, Lowe B. The patient health questionnaire somatic, anxiety, and depressive symptom scales: a systematic review. Gen Hosp Psychiatry. 2010;32:345-359.

21. Kroenke K, Spitzer RL, Williams JB. The PHQ-9: validity of a brief depression severity measure. J Gen Intern Med. 2001;16:606-613.

22. Unutzer J, Katon WJ, Fan MY, et al. Long-term cost effects of collaborative care for late-life depression. Am J Manag Care. 2008;14:95-100.

23. Katon WJ, Lin EH, Von Korff M, et al. Collaborative care for patients with depression and chronic illnesses. N Engl J Med. 2010;363(27):2611-2620.

Address correspondence to:

Peter M. McGough, MD

UW Medicine Neighborhood Clinics, and Department of Family Medicine 4333 Brooklyn Ave NE, Seattle WA 98195

Campus Box: 359410

E-mail: pmcgough@uwpn.org 